\title{
Walking epidurals for labour analgesia: do they benefit anyone?
}

\author{
Roanne Preston, MD
}

Published online: 6 January 2010

(C) Canadian Anesthesiologists' Society 2009

The "walking epidural" first appeared in the early 1990s. In some ways, it tested our widely held beliefs about how to provide effective and safe labour epidural analgesia and responded to women's requests to have effective labour analgesia without being confined to bed. In the first versions of the walking epidural, the combined spinalepidural (CSE) technique was used. It provided initial analgesia with intrathecal narcotic, which was followed by a more standard continuous epidural infusion. ${ }^{1,2}$ This novel technique challenged our assumptions about the amount of neuraxial medication needed to initiate and maintain labour analgesia. Many labour analgesia studies ensued that resulted in significant changes to both the technique and the dosing strategies, and they led to a deeper understanding of the way in which local anesthetics and opioids work alone and synergistically in the neuraxium. Critical assessment of ambulation in labour with an epidural in situ has provided enormous benefit to women in labour, has changed how anesthesiologists provide labour epidural analgesia, and has added to the body of literature to convince our skeptical colleagues in obstetrics that epidurals don't necessarily mean an operative delivery. The end result of this scientific activity is patient-controlled labour epidural analgesia with low-dose solutions-there are so many benefits in that simple phrase. So, why has the enthusiasm for walking epidurals faded?

R. Preston, MD $(\varangle)$

Department of Anesthesia, BC Women's Hospital and Health Centre, Rm 1Q72, 4500 Oak Street, Vancouver, BC V6H 3N1, Canada

e-mail: rpreston@cw.bc.ca

R. Preston, MD

Department of Anesthesiology, Pharmacology and Therapeutics, University of British Columbia, Vancouver, BC, Canada
While it is true that ambulation per se has not been shown to alter labour outcome, ${ }^{3-7}$ maintaining mobility appeals to women although they may be at the point of requiring neuraxial analgesia. The fact that they may not take full advantage of this opportunity does not negate its other benefit of minimizing motor block. It is also true that the "mobile mom" can create more work for staff; however, once anesthesiologists establish management and safety protocols for ambulation, the added work belongs to the nurse and/or the midwife, many of whom support maintaining mobility. ${ }^{8}$ Safety of the perambulating parturient with an epidural has been established-her balance is just as good as any pregnant woman's is at term, ${ }^{9,10}$ blood pressure is possibly more stable than in the woman who remains lying in bed $^{11}$ and the fetus probably benefits from complete absence of aortocaval compression. $^{12}$ So, why has the movement to mobility stalled? Have anesthesiologists lost their taste for the CSE because of fetal bradycardia and maternal pruritus? ${ }^{13}$ While CSEs opened the door to mobility, it has been clearly proven that low-dose epidurals with no intrathecal component work incredibly well and allow for safe ambulation. ${ }^{14,15}$

Perhaps we should reflect on the reason this "fad" started. Standard (aka dense) epidural analgesia did not always lead to maternal satisfaction with the analgesia because of the sense of loss of body control and the increased need for an instrumental vaginal delivery, especially if the mother was primiparous. ${ }^{16}$ So, intrepid obstetrical anesthesiologists began to challenge the status quo and experiment with the techniques of using intrathecal lipid soluble narcotics for initial analgesia, progressively more dilute solutions in the epidural space for subsequent analgesia, and then applying patient-controlled analgesia technology. Many studies used the ability to ambulate as one of the required outcomes, as it 
is linked to maternal satisfaction. All of this advanced the way anesthesiologists approached labour epidural analgesia, causing us to think beyond the simple mindset of "painless labour equals good labour". Do we believe that several hours of bed rest in the somewhat awkward wedged supine position after epidural placement will preserve normal labour physiology and the likelihood of a spontaneous vaginal delivery (SVD)? If we do, we need a refresher course in labour physiology and perhaps even gravity.

While all of the anesthesia research initiatives on whether ambulation can improve labour outcome in women with epidurals have not been positive, counter studies in nursing and midwifery journals have shown that the upright position in first stage and "anything but lithotomy" in second stage is beneficial to achieving a SVD. ${ }^{7,17-19}$ Upright position in first stage is associated with less pain, better uterine contractions, and shortened duration of labour. ${ }^{7}$ A recently published study showed that ambulation in labour with an epidural allows women to void spontaneously and reduces the need for urinary catheterization. ${ }^{20}$ And of course there is that maternal satisfaction "thing"-low-dose mobile epidurals are superior to traditional techniques in this respect, some due to the reduced rate of assisted vaginal delivery. ${ }^{16}$ Some anesthesiologists may shrug their shoulders at these kinds of benefits, but they are significant for the parturient. Why wouldn't we want to see shorter less painful labours and less need for vacuum or forceps? After all, labour epidural analgesia should have the least impact as possible on normal labour physiology - this is a basic tenet of ideal labour analgesia.

Perhaps we have become complacent, thinking we have moved successfully to using low-dose epidurals, patientcontrolled no less, and the debate about epidurals and Cesarean delivery rates appears to have abated. In the published studies, most women on ambulation did not actually take the opportunity to walk-40 to $60 \mathrm{~min}$ at most during the remainder of first stage once the epidural was sited. At the time these studies were carried out, our nursing colleagues still had a lot of trepidation about the technique, and perhaps they did not truly encourage walking. Now, many of them believe that maintaining mobility in labour has benefits to the parturient as well as to their own back health. We should not be the hindrance to allowing for mobility.

What does this mean to the average anesthesiologist providing labour epidural analgesia services? First, our departments should have up-to-date policies in place that allow women mobility after receiving a labour epidural and, indeed, to walk if they so choose. Second, we should use the scientific information generated from the walking epidural "fad" and use dilute solutions of local anesthetic and opioid as the standard of care, preferably in a patientcontrolled mode, which leads to even less local anesthetic consumption and hence less motor block over the duration of labour. ${ }^{21}$ And finally, we should support nurses and midwives in their desire to promote "normal birth" (It doesn't mean unmedicated!). Be an advocate in your department for "mobile labour epidural analgesia"- the labouring woman will love you for it!

Is the walking epidural fading into the annals of obstetric anesthesia history? It shouldn't - the benefits gained since introducing the technique remain. It may be the moniker that needs to change. Mobile labour epidural analgesia can put an end to the wedged supine lithotomy mindset that is synonymous with interventional childbirth practices. The perambulating parturient (with an epidural) may remain a rare sight, but if she is getting up in her labour room, voiding spontaneously, and trying out more physiologic delivery positions, then she has benefitted from the research that has been done since the phrase "walking epidural" was created.

\section{Péridurales ambulatoires pour l'analgésie du travail obstétrical: à qui profitent-elles?}

$\mathrm{La}$ «péridurale ambulatoire » a fait son apparition au début des années 90. D’une certaine manière, cette technique a mis à l'épreuve nos croyances très répandues quant à la façon de procurer une analgésie péridurale pour le travail qui soit à la fois efficace et sécuritaire; en outre, elle a répondu à la demande des parturientes désirant recevoir une analgésie du travail efficace sans pour autant être confinées au lit. Les premières versions de la péridurale ambulatoire utilisaient une technique d'analgésie péridurale-rachidienne combinée. Cette méthode procurait l'analgésie de départ à l'aide d'un opioïde intrathécal, suivi d'une perfusion péridurale continue plus standardisée. ${ }^{1,2}$ Cette technique innovante a ébranlé nos préjugés concernant la quantité de médication neuraxiale nécessaire à installer et maintenir une analgésie pour le travail. Nombre d'études portant sur l'analgésie pour le travail obstétrical ont ensuite vu le jour, lesquelles ont généré des changements considérables aussi bien au niveau de la technique que des stratégies de dosage, menant à une meilleure compréhension de la façon dont les anesthésiques locaux et les opioïdes agissent seuls ou en synergie au niveau neuraxial. L'évaluation critique de l'ambulation avec une péridurale in situ pendant le travail a offert des bienfaits énormes aux femmes en travail et changé la manière dont les anesthésiologistes procurent l'analgésie péridurale pour le travail. Ces données se sont ajoutées à la littérature et sont de nature à convaincre nos collègues obstétriciens sceptiques que péridurale ne rime pas toujours avec 
accouchement par césarienne. Au final de toute cette activité scientifique: une analgésie péridurale pour le travail contrôlée par la patiente avec des solutions à faible dose - que de bienfaits dans cette simple expression! Dès lors, pourquoi l'enthousiasme pour les péridurales ambulatoires est-il retombé?

Il est vrai qu'il n'a pas été démontré que l'ambulation changeait en soi le pronostic du travail ${ }^{3-7}$; toutefois, le maintien de la mobilité est attrayant pour les femmes, même lorsqu'elles sont sur le point de demander une analgésie neuraxiale. Le fait qu'elles pourraient ne pas profiter pleinement de cette possibilité ne nie pas l'autre bienfait de ce type d'analgésie, à savoir la minimisation du bloc moteur. Mais il est vrai aussi qu'une «mère mobile » peut créer plus de travail pour le personnel soignant; cependant, dès que l'anesthésiologiste a déterminé les protocoles de prise en charge et de sécurité nécessaires à l'ambulation, le travail supplémentaire revient à l'infirmière et/ou la sage-femme, et ces dernières sont nombreuses à se ranger du côté du maintien de la mobilité. ${ }^{8}$ Dès que la sécurité de la parturiente ambulante bénéficiant d'une péridurale a été établie, son équilibre est tout aussi bon que celui de la femme enceinte normale arrivée à terme. ${ }^{9,10}$ En fait, la tension artérielle d'une parturiente qui se déplace est possiblement plus stable que celle d'une patiente qui demeure alitée, ${ }^{11}$ et le fœetus bénéficie sans doute de l'absence complète de compression aorto-cave. ${ }^{12}$ Ainsi, pourquoi le mouvement vers la mobilité a-t-il perdu son élan? Les anesthésiologistes ont-ils perdu leur goût pour l'analgésie péridurale-rachidienne combinée en raison des risques de bradycardie fotale et de prurit maternel ? $^{13}$ Bien que l'analgésie péridurale-rachidienne combinée ait ouvert la voie à la mobilité, il a été clairement prouvé que les péridurales à faible dose sans composante intrathécale sont très efficaces et permettent une ambulation sécuritaire. ${ }^{14,15}$

Il nous faut peut-être réfléchir à la raison qui se cache à l'origine de cette «mode ». L'analgésie péridurale standard (autrement dit, 'dense') n'a pas toujours été considérée satisfaisante par les parturientes en raison du sentiment de perte de contrôle sur leur corps et du besoin accru d'accouchement vaginal opératoire, particulièrement chez les primipares. ${ }^{16}$ Pour cette raison, certains anesthésiologistes obstétricaux intrépides ont commencé à remettre en question le statu quo et à tester différentes techniques, notamment l'utilisation d'opioïdes liposolubles intrathécaux pour l'analgésie initiale, de solutions de plus en plus diluées dans l'espace péridural pour l'analgésie subséquente, puis le recours à une analgésie contrôlée par la patiente. Plusieurs études ont défini la capacité d'ambulation comme l'un des critères d'évaluation, étant donné que cette capacité est liée à la satisfaction maternelle. C'est ainsi que la façon dont les anesthésiologistes abordent l'analgésie péridurale pour le travail a évolué, ce qui nous a permis de penser au-delà du principe simpliste de « travail sans douleur égal bon travail obstétrical ». Croyons-nous que plusieurs heures d'alitement dans une position quelque peu inconfortable semi-couchée après la mise en place de la péridurale préserveront une physiologie de travail normale aussi bien que la probabilité d'un accouchement vaginal spontané? Si c'est le cas, il nous faut suivre d'urgence un cours de recyclage sur la physiologie du travail - et peut-être même sur la loi de la gravité.

Il est vrai que les initiatives de recherche en anesthésie sur l'amélioration du pronostic du travail obstétrical chez les femmes bénéficiant d'une péridurale grâce à l'ambulation n'ont pas toutes eu des résultats positifs. Cependant, des études opposées dans des revues spécialisées destinées aux infirmières et aux sages-femmes ont montré que la position debout durant la première phase et « tout sauf la lithotomie » durant la deuxième phase du travail favorisent un accouchement vaginal spontané..$^{717-19} \mathrm{La}$ position debout durant la première phase du travail est associée à moins de douleur, de meilleures contractions utérines, et une durée raccourcie du travail. ${ }^{7}$ Une étude récemment publiée a montré que l'ambulation pendant le travail avec une péridurale permettait aux femmes d'aller spontanément à la selle et réduisait le besoin de cathétérisation urinaire. ${ }^{20} \mathrm{Et}$, bien sûr, il y a également la satisfaction maternelle: la péridurale ambulatoire à faible dose est supérieure aux techniques traditionnelles à cet égard, en partie grâce au taux réduit d'accouchement vaginal opératoire. ${ }^{16}$ Pour certains anesthésiologistes, cette sorte d'avantages peuvent paraître dérisoires, mais ils sont considérables pour la parturiente. N'avons-nous pas envie de voir moins de douleur dans le travail obstétrical et moins de cas nécessitant le recours à la succion et aux forceps? Après tout, l'analgésie péridurale pour le travail obstétrical devrait avoir le moins de répercussions possibles sur la physiologie du travail - il s'agit là d'un principe de base pour une analgésie du travail idéale.

Peut-être sommes-nous devenus trop confiants depuis que nous sommes parvenus à administrer des péridurales à faible dose, qui plus est contrôlées par la patiente; du coup, le débat sur la péridurale et sur les taux d'accouchement par césarienne semble s'être enlisé. Dans les études publiées, la plupart des femmes en ambulation n'ont en fait pas saisi l'occasion de marcher, et celles qui l'ont saisi ont marché au maximum 40 à 60 minutes pendant le reste de la première phase du travail une fois la péridurale installée. $\mathrm{Au}$ moment où ces études étaient réalisées, nos collègues des soins infirmiers étaient encore très anxieux quant à cette technique, et il se peut qu'ils n'aient pas véritablement encouragé les patientes à marcher. Aujourd'hui, nombre de ces collègues pensent que le maintien de la mobilité pendant le travail présente des avantages pour la parturiente aussi 
bien que pour leur propre santé dorsale. Nous ne devrions pas être le frein à la mobilité.

Quelles sont les implications de cette déclaration pour l'anesthésiologiste moyen qui offre des services d'analgésie péridurale pour le travail? En premier lieu, nos départements devraient disposer de politiques à jour qui autorisent la mobilité des parturientes une fois qu'elles ont reçu une péridurale pour le travail, et qui leur permette même de marcher si tel est leur souhait. Deuxièmement, nous devrions nous servir des informations scientifiques engendrées par la «mode » de la péridurale ambulatoire et utiliser des solutions diluées d'anesthésique local et d'opioïde comme norme de soin, de préférence dans un mode contrôlé par la patiente, ce qui mène à une consommation encore plus réduite d'anesthésique local et par là même à un bloc moteur moindre pendant la durée du travail obstétrical. ${ }^{21}$ Dernièrement, nous devrions soutenir les infirmières et les sages-femmes dans leur désir de promouvoir des «naissances normales» (ce qui ne veut pas dire sans médication aucune!). Faites-vous le défenseur de « l'analgésie péridurale mobile pour le travail» dans votre département - et les parturientes en travail vous en remercieront!

La péridurale ambulatoire est-elle en train de se perdre dans les annales de l'histoire de l'anesthésie obstétricale? Elle ne devrait pas, parce que les bienfaits acquis depuis l'introduction de cette technique demeurent. Le sobriquet de cette technique est peut-être ce qu'il faut changer. L'analgésie péridurale mobile pour le travail a le pouvoir de mettre fin à la philosophie de lithotomie dorsale qui va actuellement de pair avec les techniques d'accouchement opératoire. Il se peut qu'il soit toujours aussi rare de voir une parturiente qui marche (avec une péridurale), mais si elle se lève dans sa salle de travail, va spontanément à la selle et essaie d'autres positions d'accouchement physiologique, alors elle a tiré profit des recherches menées depuis que le terme «péridurale ambulatoire » a été inventé.

Conflicts of interest None declared.

\section{References}

1. Collis RE, Baxandall ML, Srikantharajah ID, Edge G, Kadim $M Y$, Morgan BM. Combined spinal epidural analgesia with ability to walk throughout labour. Lancet $1993 ; 341: 767-8$.

2. Camann W, Abouleish A. Spinal epidural analgesia and walking throughout labour. Lancet 1993; 341: 1095.

3. Wilson MJ, MacArthur C, Cooper GM, Shennan A, COMET Study Group UK. Ambulation in labour and delivery mode: a randomised controlled trial of high-dose vs mobile epidural analgesia. Anaesthesia 2009; 64: 266-72.
4. Collis RE, Harding SA, Morgan BM. Effect of maternal ambulation on labour with low-dose combined spinal-epidural analgesia. Anaesthesia 1999; 54: 535-9.

5. Roberts $C L$, Algert CS, Olive E. Impact of first-stage ambulation on mode of delivery among women with epidural analgesia. Aust NZ J Obstet Gynaecol 2004; 44: 489-94.

6. Vallejo MC, Firestone LL, Mandell GL, Jaime F, Makishima S, Ramanathan $S$. Effect of epidural analgesia with ambulation on labor duration. Anesthesiology 2001; 95: 857-61.

7. Lawrence A, Lewis L, Hofmeyr GJ, Dowswell T, Styles C. Maternal positions and mobility during first stage labour. Cochrane Database Syst Rev 2009; (2): CD003934.

8. Mayberry LJ, Strange LB, Suplee PD, Gennaro S. Use of upright positioning with epidural analgesia: findings from an observational study. MCN Am J Matern Child Nurs 2003; 28: 152-9.

9. Davies J, Fernando R, McLeod A, Verma S, Found P. Postural stability following ambulatory regional analgesia for labor. Anesthesiology 2002; 97: 1576-81.

10. Pickering AE, Parry MG, Ousta B, Fernando R. Effect of combined spinal-epidural ambulatory labor analgesia on balance. Anesthesiology 1999; 91: 436-41.

11. Shennan A, Cooke V, Lloyd-Jones F, Morgan B, de Swiet M. Blood pressure changes during labour and whilst ambulating with combined spinal epidural analgesia. Br J Obstet Gynaecol 1995; 102: 192-7.

12. al-Mufti R, Morey R, Shennan A, Morgan B. Blood pressure and fetal heart rate changes with patient-controlled combined spinal epidural analgesia while ambulating in labour. $\mathrm{Br} \mathrm{J}$ Obstet Gynaecol 1997; 104: 554-8.

13. Van de Velde M, Teunkens A, Hanssens $M$, Vandermeersch E, Verhaeghe $J$. Intrathecal sufentanil and fetal heart rate abnormalities: a double-blind, double placebo-controlled trial comparing two forms of combined spinal epidural analgesia with epidural analgesia in labor. Anesth Analg 2004; 98: 1153-9.

14. Wilson MJ, Cooper G, MacArthur C, Shennan A, Comparative Obstetric Mobile Epidural Trial (COMET) Study Group UK. Randomized controlled trial comparing traditional with two "mobile" epidural techniques: anesthetic and analgesic efficacy. Anesthesiology 2002; 97: 1567-75.

15. Simmons SW, Cyna AM, Dennis AT, Hughes D. Combined spinalepidural versus epidural analgesia in labour. Cochrane Database Syst Rev 2007; (3): CD003401.

16. Comparative Obstetric Mobile Epidural Trial (COMET) Study Group UK. Effect of low-dose mobile versus traditional epidural techniques on mode of delivery: a randomised controlled trial. Lancet 2001; 358: 19-23.

17. Bodner-Adler B, Bodner K, Kimberger O, Lozanov P, Husslein P, Mayerhofer $K$. Women's position during labour: influence on maternal and neonatal outcome. Wien Klin Wochenschr 2003; 115: 720-3.

18. Gupta JK, Nikodem VC. Woman's position during second stage of labour. Cochrane Database Syst Rev 2000; (2): CD002006.

19. Roberts $C L$, Algert CS, Cameron CA, Torvaldsen S. A metaanalysis of upright positions in the second stage to reduce instrumental deliveries in women with epidural analgesia. Acta Obstet Gynecol Scand 2005; 84: 794-8.

20. Weiniger CF, Yaghmour $H$, Nadjari $M$, et al. Walking reduces the post-void residual volume in parturients with epidural analgesia for labor: a randomized-controlled study. Acta Anaesthesiol Scand 2009; 53: 665-72.

21. van der Vyver M, Halpern S, Joseph G. Patient-controlled epidural analgesia versus continuous infusion for labour analgesia: a meta-analysis. Br J Anaesth 2002; 89: 459-65. 